\title{
Decay of turbulence generated by a square-fractal-element grid
}

\author{
R. J. Hearst and P. Lavoie $\dagger$ \\ Institute for Aerospace Studies, University of Toronto, Toronto, ON M3H 5T6, Canada \\ (Received ?; revised ?; accepted ?. - To be entered by editorial office)
}

A novel square-fractal-element grid was designed in order to increase the downstream measurement range of fractal grid experiments relative to the largest element of the grid. The grid consists of a series of square-fractal-elements mounted to a background mesh with spacing $L_{0}=100 \mathrm{~mm}$. Measurements were performed in the region $3.5 \leqslant x / L_{0} \leqslant$ 48.5 , which represents a significant extension to the $x / L_{0}<20$ of previously reported square fractal grid measurements. For the region $x / L_{0} \gtrsim 24$ it was found that a powerlaw decay region following $\left\langle q^{2}\right\rangle \sim\left(x-x_{0}\right)^{m}$ exists with decay exponents of $m=-1.39$ and -1.37 at $R e_{L_{0}}=57,000$ and 65,000 , respectively. This agrees with decay values previously measured for regular grids $(-1 \gtrsim m \gtrsim-1.4)$. The turbulence in the near-grid region, $x / L_{0}<20$, is shown to be inhomogeneous and anisotropic, in apparent contrast with previous fractal grid measurements. Nonetheless, power-law fits to the decay of turbulent kinetic energy in this region result in $m=-2.79$, similar to $m \approx-2.5$ recently reported by Valente \& Vassilicos (2011) for space-filling square fractals. It was also found that $C_{\epsilon}$ is approximately constant for $x / L_{0} \geqslant 25$, while it grows rapidly for $x / L_{0}<20$. These results reconcile previous fractal-generated turbulence measurements with classical grid turbulence measurements.

Key words: (to be entered by typesetter)

\section{Introduction}

The decay of turbulence downstream of a grid in a wind tunnel is often used as an experimental benchmark for the evaluation of turbulence theories and models since it provides an empirical approximation of homogeneous, isotropic turbulence (HIT). HIT is governed by the von Kármán-Howarth equation (von Kármán \& Howarth 1938), which predicts that the decay of turbulent kinetic energy should follow a power-law, viz.

$$
\left\langle q^{2}\right\rangle \sim\left(t-t_{0}\right)^{m}
$$

where $\left\langle q^{2}\right\rangle=\left\langle u^{2}\right\rangle+\left\langle v^{2}\right\rangle+\left\langle w^{2}\right\rangle$ is twice the turbulent kinetic energy, $t$ is time, and $t_{0}$ is a virtual origin. Different theoretical solutions predict that the power-law exponent may take on one of several values, e.g. $m=-6 / 5$ (Saffman 1967), and $m=-10 / 7$ (Batchelor 1948). Since the seminal work of Comte-Bellot \& Corrsin (1966), who used multiple grids to determine the value of $m$, exponent values over the range $-1 \gtrsim m \gtrsim-1.4$ have been reported (Gad-el Hak \& Corrsin 1974; Mohamed \& LaRue 1990; Lavoie et al. 2007; Krogstad \& Davidson 2010, 2011). Given the empirical variation of $m$, George (1992) developed an updated solution to the von Kármán-Howarth equation that allowed $m$ to be dependent on initial conditions and suggested $m \rightarrow-1$ as $R e_{\lambda} \rightarrow \infty$.

\footnotetext{
$\dagger$ Email address for correspondence: lavoie@utias.utoronto.ca
} 
Recently, the work of Hurst \& Vassilicos (2007) has catalyzed a modern resurgence in grid turbulence research (Mazellier \& Vassilicos 2010; Valente \& Vassilicos 2011, 2012; Krogstad \& Davidson 2010, 2011, 2012). The decay behind the space-filling square fractals of Hurst \& Vassilicos (2007) was found to be very rapid, and was originally believed to follow an exponential law. Later, this hypothesis was updated by Valente \& Vassili$\cos$ (2011), who showed that the turbulence behind this type of grid does in fact decay according to a power-law, but one with a high decay rate, $m \approx-2.5$. Furthermore, they observed that the streamwise integral length scale was approximately proportional to the Taylor microscale, i.e. $L_{u} / \lambda \approx$ constant, and that the turbulence may be described by a single-length-scale, as normalization by large scale variables collapsed the spectra at all wavenumbers (which is atypical of grid turbulence experiments). Vassilicos and coworkers have linked this form of rapid decay to a possible breakdown of the traditional dissipation scaling, $\epsilon \sim\left\langle q^{2}\right\rangle^{3 / 2} / L_{u}$. Ultimately, these variations from previous grid turbulence results are thought to be associated with the lasting influence of the 'multi-scale' initial conditions induced by the fractal geometry.

To date, fractal studies have employed 'space-filling' square fractals where a single fractal element occupies the entire wind tunnel cross-section. In such a setup, the largest element of the grid, with length $L_{0}$, is approximately half the tunnel height, $H$, i.e. $H / L_{0}<2$. Corrsin (1963) states that for reasonable homogeneity, $H / M \gg 1$, where $M$ is the mesh length of the grid. If the largest scale induced by the grid is of order $L_{0}$, making it roughly equivalent to $M$, then Corrsin's criterion is not satisfied. This may have a negative impact on the flow's approximation of HIT (Wang \& George 2002). Furthermore, given the size of $L_{0}$, the relative downstream distance was limited $\left(x / L_{0}<20\right)$ in previous studies.

In order to reconcile the rapid decay results of Valente \& Vassilicos (2011) with previous measurements, Krogstad \& Davidson (2011) designed and tested multi-scale 'cross-grids' that consisted of a regular square mesh with alternating bars of different thickness. Their results demonstrated that the cross-grid multi-scale turbulence did decay with traditional values between $-1.12 \geqslant m \geqslant-1.25$. Later, Krogstad \& Davidson (2012) performed measurements in the near-grid region of cross-grids and discovered that the flow was similar to that produced downstream of a square fractal. Valente \& Vassilicos (2012) have also shown that at sufficiently high Reynolds numbers, regular grids can manifest some of the characteristics demonstrated by the square fractals. However, previous work has not specifically addressed how square fractal-generated turbulence behaves in the far-field, which is particularly significant given the earlier measurements of Hurst \& Vassilicos (2007) that showed significantly different streamwise evolution of the turbulence downstream of square fractals compared to cross-grids. In fact, Valente \& Vassilicos (2011) suggest that if a multi-scale grid was designed such that the wakes of the various elements could interact in a relatively narrow range, citing specifically cross-grids, that it is likely that the produced turbulence would be similar to that produced by a regular grid.

A new design strategy, where a small square fractal element is repeated several times in a homogeneous mosaic pattern, is implemented in the present work to increase $H / L_{0}$. This strategy both improves the transverse homogeneity and extends the downstream range relative to $L_{0}$, addressing the limitations of earlier fractal work while preserving the fractal geometry. As such, both the near-grid region, overlapping with previous fractal experiments, and the far-field of the fractal geometry may be investigated. 


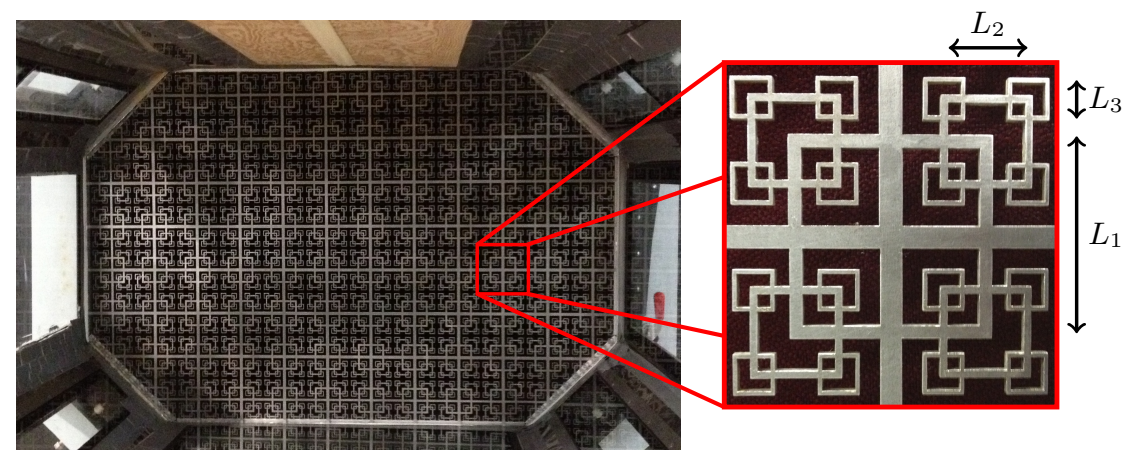

FIGURE 1. Square-fractal-element grid positioned in situ (left) with a blow-up of a single fractal element (right) and labels defining dimensions.

\section{Experimental procedure}

\subsection{The square-fractal-element grid}

A new type of square-fractal-element grid was designed in order to perform measurements farther downstream than previously attainable with the space-filling square fractal geometry (see figure 1). In this design, several $N=3$ square-fractal-elements (where $N$ is the number of times the fractal shape is repeated) were mounted to a background grid with mesh length $M=L_{0}=100 \mathrm{~mm}$ and thickness $t_{0}=6.7 \mathrm{~mm}$. The fractal elements have lengths $L_{i}=55.6,24.7$, and $11.0 \mathrm{~mm}$ with thicknesses $t_{i}=4.1,2.5$, and $1.5 \mathrm{~mm}$. The factors by which the length and thickness were incremented for each fractal iteration were $R_{L}=2.25$ and $R_{t}=1.65$, respectively. The solidity of the grid is $\sigma=0.39$. The grid was laser cut out of a single sheet of $3 \mathrm{~mm}$ thick stainless steel.

\subsection{Data acquisition}

Measurements were performed in the low-speed closed-loop wind tunnel at the University of Toronto Institute for Aerospace Studies. The wind tunnel has a background turbulence intensity of $0.05 \%$ for $U \leqslant 13 \mathrm{~m} / \mathrm{s}$. The test-section is $5 \mathrm{~m}$ long and has a cross-sectional area of $1.2 \mathrm{~m} \times 0.8 \mathrm{~m}$. The corners of the test-section are adjustable and were used to create a zero pressure gradient over its length, such that the mean velocity, $U$, was maintained to within $\pm 1 \%$ throughout the latter two-thirds of the test-section. Far downstream of the grid, $x / L_{0} \geqslant 23$, tests were conducted at two Reynolds numbers, $R e_{L_{0}}=U L_{0} / \nu=57,000$ and 65,000. For the higher $R e_{L_{0}}$, measurements were also conducted in the near-field of the grid, beginning at $x / L_{0}=3.5$. Constant temperature anemometry was utilized to perform measurements of the velocity fluctuations. Two different anemometers were used for the tests in order to both ensure consistency of results, and improve temporal resolution close to the grid. Experimental setup details are provided in table 1 . Data were acquired with both a single-wire and X-wires simultaneously; the single-wire was used to improve the spatial resolution of the measurement of $u$. The hot-wires were prepared in-house using $2.5 \mu \mathrm{m}$ thick copper-coated tungsten wire mounted to Dantec-style prongs. Wire sensing lengths, $\ell$, are provided in table 1 . All sensing lengths were maintained to $\pm 0.05 \mathrm{~mm}$. The separation between the $\mathrm{X}$-wires was $1.1 \mathrm{~mm}$. The single-wire and X-wire were separated laterally by $4 \mathrm{~mm}$ $=0.04 L_{0}$. Resolution estimates are provided in table 1 , relating the wire length, $\ell$, to the Kolmogorov length scale, $\eta=\nu^{3 / 4} /\langle\epsilon\rangle^{1 / 4}$, where $\langle\epsilon\rangle$ is the mean dissipation rate of turbulent kinetic energy (hereafter referred to simply as dissipation). The hot-wires were calibrated in situ at the farthest downstream location, where the turbulence in- 


\begin{tabular}{cccccccc}
\hline $\begin{array}{c}U \\
{[\mathrm{~m} / \mathrm{s}]}\end{array}$ & $R e_{L_{0}}$ & Anemometer & $\begin{array}{c}f_{s} \\
{[\mathrm{kHz}]}\end{array}$ & $\begin{array}{c}f_{c} \\
{[\mathrm{kHz}]}\end{array}$ & $\begin{array}{c}\ell \\
{[\mathrm{mm}]}\end{array}$ & $\begin{array}{c}\text { Resolution } \\
{[\ell / \eta]}\end{array}$ & $\begin{array}{c}\text { Meas. range } \\
{\left[x / L_{0}\right]}\end{array}$ \\
\hline 9.0 & 57,000 & Newcastle & 18.5 & 9.2 & 0.65 & {$[1.5,2.4]$} & {$[23.7,44.6]$} \\
10.6 & 65,000 & Dantec 56C & 30.0 & $14.5,10.5$ & 0.55 & {$[1.4,5.6]$} & {$[3.5,48.5]$}
\end{tabular}

TABLE 1. Experimental setup details. See Miller et al. (1987) for details on the University of Newcastle anemometer.

tensity was less than $2 \%$. The single-wire was calibrated with ten velocities fit with a fourth-order polynomial. The X-wires were calibrated with ten velocities and seven angles, using the look-up table approach described by Burattini \& Antonia (2005). Pre- and post-calibrations were performed for every set of streamwise measurements to account for hot-wire drift. The temperature correction methodology of Hultmark \& Smits (2010) was employed, although the test-section temperature was maintained to $\pm 0.5{ }^{\circ} \mathrm{C}$ for each test. Samples were recorded for 8 minutes or longer in order to ensure better than $\pm 1 \%$ convergence of $\left\langle q^{2}\right\rangle$ based on the $95 \%$ confidence interval (Benedict \& Gould 1996); $\left\langle q^{2}\right\rangle$ was estimated from $\left\langle q^{2}\right\rangle=\left\langle u^{2}\right\rangle+2\left\langle v^{2}\right\rangle$ after verifying $\left\langle v^{2}\right\rangle \approx\left\langle w^{2}\right\rangle$. In the streamwise direction, measurements were performed in $35 \mathrm{~mm}\left(\approx L_{0} / 3\right)$ increments in the range $0.35 \mathrm{~m} \leqslant x \leqslant 4.85 \mathrm{~m}\left(3.5 \leqslant x / L_{0} \leqslant 48.5\right)$ using a stepper-motor-actuated traverse. The streamwise scan was performed along $(y, z)=(30 \mathrm{~mm}, 0)$, where $(y, z)=(0,0)$ is the centre of the test-section and corresponds to the centre of an opening in the background grid. This location was chosen because it resulted in a constant mean velocity over the majority of the tunnel length. Horizontal profiles were also measured at several downstream locations over the range $-26 \mathrm{~cm} \leqslant y \leqslant 21 \mathrm{~cm}\left(-2.6 \leqslant y / L_{0} \leqslant 2.1\right)$ in increments of $30 \mathrm{~mm}$ along the $z=0$ axis.

\subsection{Data post-processing}

Post-acquisition, corrections were applied to minimize high- and low-frequency noise, account for the finite spatial resolution of the hot-wire probes, and compensate for the filtering inherent to numerical differentiation of a discrete signal. First, the data is recursively band-pass filtered between $0.75 \mathrm{~Hz}$ and the Kolmogorov frequency, $f_{K}=U / 2 \pi \eta$, using the technique proposed by Mi et al. $(2005,2011)$. During this process, $f_{K}$ is calculated and then used as $f_{c}$ for a fifth-order digital Butterworth filter. The Kolmogorov frequency is then recalculated, and the data filtered again with the new $f_{K}$. The process is repeated iteratively until $\left|f_{K}-f_{K}^{\text {old }}\right| \leqslant 10^{-3}$. Convergence is typically reached in 4 or fewer iterations.

Corrections for the finite spatial resolution of the hot-wire probes were made using the approach of Wyngaard (1968) for the single-wire, and the extension proposed by Zhu \& Antonia $(1995 a, 1996)$ for the X-wire. This technique employs a modelled 3dimensional energy spectrum, $E(k)$, the measured estimate of $\eta$, and the finite length of the sensor to determine a priori the difference between the measured and theoretical velocity spectra. Here, we use the model spectrum of Meyers \& Meneveau (2008) that is $R e_{\lambda}$ dependent and accurately models the low wavenumber behaviour. Gradients are estimated using Taylor's hypothesis and a sixth-order centred-difference scheme, which has been shown to increase the accuracy of gradient estimates when compared to the use of lower-order schemes (Hearst et al. 2012). Corrections for the gradients are applied as per Hearst et al. (2012). While the aforementioned corrections assume homogeneity and 


\begin{tabular}{rccccccccc}
\hline$x / L_{0}$ & 10.2 & 15.1 & 19.9 & 24.9 & 30.1 & 35.4 & 39.9 & 44.8 & \\
& & & & & & & & & \\
\hline$e_{\lambda, \text { iso }}$ & 167 & 126 & 106 & 97 & 88 & 83 & 80 & 77 & \\
$R e_{\lambda}$ & 152 & 118 & 101 & 94 & 89 & 86 & 84 & 81 & \\
$u^{\prime} / U$ & 7.53 & 5.20 & 3.94 & 3.20 & 2.67 & 2.34 & 2.11 & 1.90 & {$[\%]$} \\
$L_{u}$ & 22.7 & 24.3 & 26.9 & 29.4 & 30.9 & 34.8 & 36.3 & 39.0 & {$[\mathrm{~mm}]$} \\
$\lambda$ & 3.1 & 3.5 & 3.9 & 4.5 & 5.1 & 5.6 & 6.1 & 6.5 & {$[\mathrm{~mm}]$} \\
$\eta$ & 0.14 & 0.17 & 0.21 & 0.25 & 0.29 & 0.33 & 0.36 & 0.39 & {$[\mathrm{~mm}]$} \\
$\langle\epsilon\rangle_{d}$ & - & - & - & 1.14 & 0.62 & 0.38 & 0.27 & 0.19 & {$\left[\mathrm{~m}^{2} \mathrm{~s}^{-3}\right]$} \\
$\langle\epsilon\rangle_{X W}$ & 11.58 & 4.85 & 2.24 & 1.16 & 0.68 & 0.43 & 0.31 & 0.22 & {$\left[\mathrm{~m}^{2} \mathrm{~s}^{-3}\right]$} \\
$\langle\epsilon\rangle_{i s o}$ & 13.03 & 5.50 & 2.54 & 1.33 & 0.78 & 0.50 & 0.36 & 0.26 & {$\left[\mathrm{~m}^{2} \mathrm{~s}^{-3}\right]$} \\
$u^{\prime} / v^{\prime}$ & 1.30 & 1.23 & 1.19 & 1.19 & 1.18 & 1.18 & 1.18 & 1.19 & \\
$\left\langle(\partial v / \partial x)^{2}\right\rangle /\left\langle(\partial u / \partial x)^{2}\right\rangle$ & 1.65 & 1.65 & 1.66 & 1.63 & 1.63 & 1.63 & 1.61 & 1.59 & \\
$L_{u} / L_{v}$ & 2.7 & 2.5 & 2.5 & 2.5 & 2.4 & 2.5 & 2.4 & 2.4 & \\
$L_{u} / \lambda$ & 7.3 & 7.0 & 6.9 & 6.6 & 6.2 & 6.2 & 6.0 & 6.0 & \\
$\xi_{u}$ & 0.8 & 0.6 & 0.4 & 0.3 & 0.3 & 0.2 & 0.2 & 0.2 & {$[\%]$} \\
$\xi_{v}$ & 0.3 & 0.2 & 0.2 & 0.2 & 0.2 & 0.1 & 0.1 & 0.1 & {$[\%]$} \\
$\xi_{\partial u / \partial x}$ & 5.0 & 3.6 & 2.6 & 1.9 & 1.4 & 1.1 & 0.9 & 0.8 & {$[\%]$} \\
$\xi_{\partial v / \partial x}$ & 12.8 & 9.6 & 7.2 & 5.6 & 4.4 & 3.5 & 3.1 & 2.6 & {$[\%]$}
\end{tabular}

TABLE 2. Turbulent flow properties at eight downstream locations for $R e_{L_{0}}=65,000$.

isotropy, the impact of these assumptions on the estimated corrections is small since the effect of finite resolution are primarily felt at the small scales where local homogeneity and isotropy are well approximated in grid turbulence. The accuracy and limitations of this correction methodology have been extensively tested in experiments and DNS (Antonia et al. 1994; Zhu \& Antonia 1995b; Elsner \& Elsner 1996; Zhu \& Antonia 1996; Ewing \& George 2000; Hearst et al. 2012). The effect of spatial resolution is small in the present study with the corrections being less than $1 \%$ for $\left\langle u^{2}\right\rangle$ and $\left\langle v^{2}\right\rangle$. Finite resolution effects are more significant for the gradient measurements as shown in table 2 where $\xi_{\alpha}$ is the correction applied to the quantity $\alpha=u, v, \partial u / \partial x$, or $\partial v / \partial x$. The approximate measurement uncertainties are less or equal to $\pm 3 \%$ on $\left\langle u^{2}\right\rangle, \pm 4 \%$ on $\left\langle v^{2}\right\rangle, \pm 5 \%$ on $\left\langle q^{2}\right\rangle$, $\pm 8 \%$ on $\left\langle(\partial u / \partial x)^{2}\right\rangle$, and $\pm 10 \%$ on $\left\langle(\partial v / \partial x)^{2}\right\rangle$.

\subsection{Estimating the mean kinetic energy dissipation rate}

It is notoriously difficult to obtain accurate experimental estimates of $\langle\epsilon\rangle$. In grid turbulence, the most accurate means of estimating $\langle\epsilon\rangle$ is from the turbulent kinetic energy budget for HIT, viz.

$$
\langle\epsilon\rangle_{d}=-\frac{U}{2} \frac{\mathrm{d}\left\langle q^{2}\right\rangle}{\mathrm{d} x}
$$

In order to obtain an accurate estimate of the gradient in (2.1), a fit to $\left\langle q^{2}\right\rangle$ is used (which is described in detailed in section 3.2). The dissipation was also estimated using the assumption of local isotropy, viz.

$$
\langle\epsilon\rangle_{i s o}=15 \nu\left\langle\left(\frac{\partial u}{\partial x}\right)^{2}\right\rangle,
$$


and the less restrictive form when using a $\mathrm{X}$-wire, viz.

$$
\langle\epsilon\rangle_{X W}=3 \nu\left[\left\langle\left(\frac{\partial u}{\partial x}\right)^{2}\right\rangle+2\left\langle\left(\frac{\partial v}{\partial x}\right)^{2}\right\rangle\right] .
$$

Table 2 provides estimates of several parameters measured in the square-fractal-element grid-generated turbulence, including the dissipation estimated with all three techniques. In this work, all turbulent properties (e.g. $\lambda, \eta$ ) are calculated using $\langle\epsilon\rangle_{d}$ where the flow is homogeneous in transverse planes and $\langle\epsilon\rangle_{X W}$ elsewhere, as these are the most accurate means of estimating the dissipation in these regions. In table $2, R e_{\lambda, \text { iso }}=\left\langle u^{2}\right\rangle^{1 / 2} \lambda_{\text {iso }} / \nu$ (where $\lambda_{\text {iso }}$ is estimated from $\langle\epsilon\rangle_{\text {iso }}$ ) is provided alongside $R e_{\lambda}=\left\langle u^{2}\right\rangle^{1 / 2} \lambda / \nu$ for reference as the former is the most common Reynolds number cited in other works.

The systematic difference between $\langle\epsilon\rangle_{d},\langle\epsilon\rangle_{\text {iso }}$ and $\langle\epsilon\rangle_{X W}$ is within measurement uncertainty and has been previously observed for fractal and regular grids in different facilities using hot-wire anemometry (Lavoie et al. 2007; Valente \& Vassilicos 2011). It is most likely a consequence of the different bias errors involved in the measurements of these quantities combined with the measured deviations from isotropy. The deviations from local isotropy found in the present work are typical of grid turbulence, even when large scale isotropy is satisfied. For example, see $R d 44 w$ results from Lavoie et al. (2007) or, for a larger streamwise extent, the results of Antonia et al. (1998). The exact source of this deviation is not known, but it has been observed in a number of turbulent flows for which local isotropy would be expected (see for instance George \& Hussein 1991).

\section{Results}

\subsection{Homogeneity and isotropy}

The homogeneity and isotropy of the square-fractal-element grid-generated turbulence is assessed here before discussing its decay in the next section. The flow must be approximately homogeneous in the transverse plane, and should agree with the temporal (transformed into spatial) behaviour of HIT (Corrsin 1963). Figure 2 shows transverse Pitot tube measurements of the mean velocity, $U$, at several downstream locations. In the figure, all velocities are normalized by the spanwise average velocity at $x / L_{0}=25$, $\tilde{U}$. The figure shows that the grid bar wakes have a lasting influence for a significant downstream extent, as the wakes are still visible at $x / L_{0}=15$. The centre of the flow does not homogenize until $x / L_{0}=20$, where the centre $2 L_{0}$ have constant $U$ to within $\pm 1 \%$. This is of particular interest given previous fractal studies have been conducted in the region $x / L_{0}<20$, where, according to figure 2 , the flow has not fully homogenized.

Figure 3(a) shows transverse profiles of the turbulence intensity, $T_{q}=\left\langle q^{2}\right\rangle^{1 / 2} /\left(3^{1 / 2} U\right)$, measured at $x / L_{0}=10,25,35$, and 45 with hot-wires. The profiles are normalized by their mean $T_{q}$. At $x / L_{0}=10$ there are fluctuations in $T_{q}$ of $\pm 10 \%$, which contrasts with $\pm 3.5 \%$ for $x / L_{0} \geqslant 25$. Similarly, for $x / L_{0} \geqslant 25$ the global isotropy, $\sqrt{\left\langle u^{2}\right\rangle} / \sqrt{\left\langle v^{2}\right\rangle}=u^{\prime} / v^{\prime}$, is constant to within $\pm 2 \%$, with an averaged value of about 1.2 . Although ideally $u^{\prime} / v^{\prime}=$ 1 , the value measured here is homogenous across the flow field and is in good agreement with previous passive grid turbulence measurements (Comte-Bellot \& Corrsin 1966; Hurst \& Vassilicos 2007; Lavoie et al. 2007; Krogstad \& Davidson 2010, 2011). Local isotropy is satisfied approximately with $1.59 \leqslant\left\langle(\partial v / \partial x)^{2}\right\rangle /\left\langle(\partial u / \partial x)^{2}\right\rangle \leqslant 1.66$ (table 2), which agrees with values previously reported for grid-turbulence (Antonia et al. 1998; Lavoie et al. 2007; Krogstad \& Davidson 2011). These results are only shown for $R e_{L_{0}}=65,000$, but are representative of both cases. 


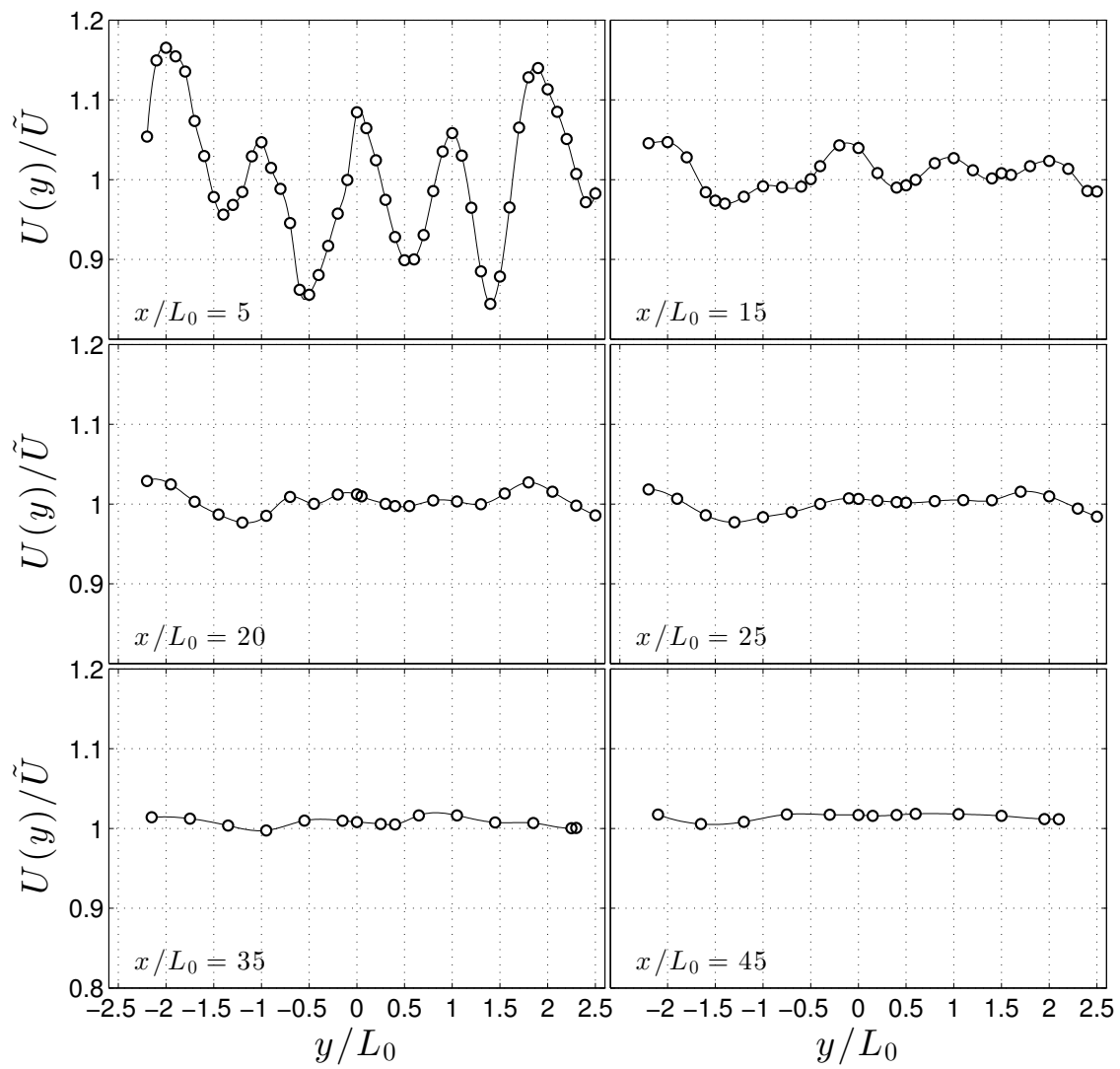

Figure 2. Transverse mean velocity, $U$, profiles at $R e_{L_{0}}=65,000$. Each profile is normalized by the spanwise average velocity at $x / L_{0}=25, \tilde{U}$.

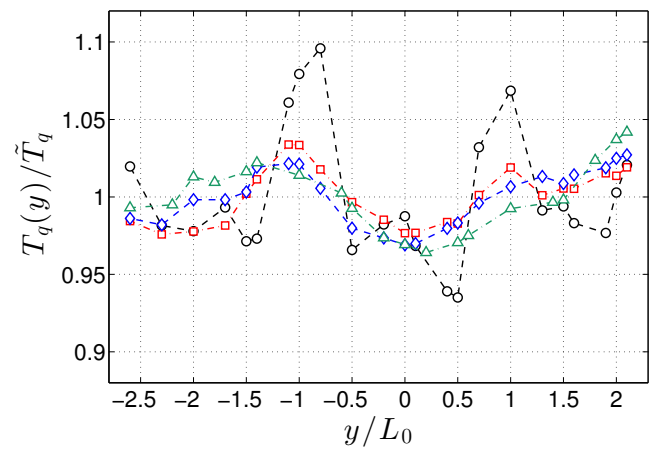

(a)

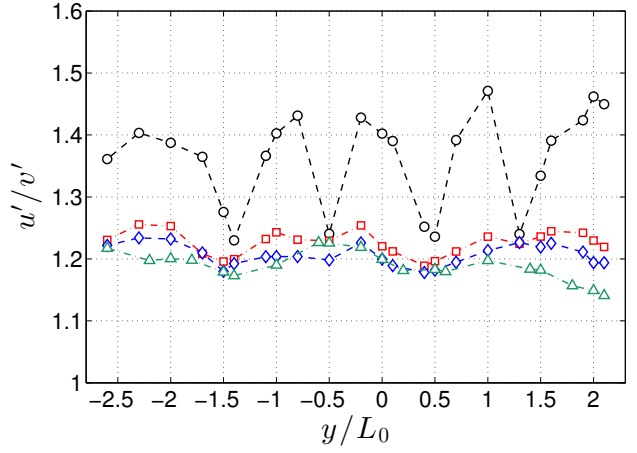

(b)

Figure 3. (a) Transverse turbulence intensity, $T_{q}=\left\langle q^{2}\right\rangle^{1 / 2} /\left(3^{1 / 2} U\right)$, profiles at $R e_{L_{0}}=65,000$. Each profile is normalized by its own mean turbulence intensity, $\tilde{T}_{q}$. (b) Transverse global isotropy, $u^{\prime} / v^{\prime}$, profiles at $R e_{L_{0}}=65,000 ;(\bigcirc) x / L_{0}=10,(\square) x / L_{0}=25,(\diamond) x / L_{0}=35,(\triangle)$ $x / L_{0}=45$. 
To further assess the streamwise homogeniety, Corrsin (1963) states that 'some necessary conditions for effective homogeneity are':

$$
\begin{aligned}
\frac{\mathrm{d} L_{u}}{\mathrm{~d} x} & \ll 1, \\
\frac{L_{u}}{\lambda} \frac{\mathrm{d} \lambda}{\mathrm{d} x} & \ll 1, \\
-\frac{L_{u}}{\left\langle u^{2}\right\rangle} \frac{\mathrm{d}\left\langle u^{2}\right\rangle}{\mathrm{d} x} & \ll 1,
\end{aligned}
$$

where $\lambda$ is the Taylor microscale and $L_{u}$ is the integral length scale based on $u$. Here, the Taylor microscale is defined as

$$
\lambda^{2}=5 \nu \frac{\left\langle q^{2}\right\rangle}{\langle\epsilon\rangle},
$$

and the integral length scale, $L_{u}$, is calculated by integrating

$$
L_{u}=\frac{1}{\left\langle u^{2}\right\rangle} \int_{0}^{r_{0}}\langle u(x) u(x+r)\rangle \mathrm{d} r,
$$

where $r_{0}$ is the first zero-crossing of the autocorrelation. Equations $(3.1 a),(3.1 b)$, and (3.1c) are plotted with offsets in figure 4 and are shown to be relatively constant and near zero for $x / L_{0}>10$.

Further assessment of the homogeneity may be made by estimating the skewness, $S_{u}$ and $S_{v}$, and flatness, $F_{u}$ and $F_{v}$, of the probability density functions of $u$ and $v$, respectively. Figure 5 demonstrates that $S_{u}(x)$ is near zero and approaches it more precisely as $x$ increases, while the transverse velocity skewness, $S_{v}(x)$, approaches zero more rapidly. Furthermore, $F_{u}(x)$ and $F_{v}(x)$ are independent of $x$ after $x / L_{0} \approx 20$ and have a mean value of $\sim 2.95$. These results for skewness and flatness are in good agreement with previous grid turbulence studies (Mohamed \& LaRue 1990; Krogstad \& Davidson 2010, 2011).

Finally, the ratio $L_{u} / L_{v}$ for isotropic turbulence is 2 . In the present experiments, this ratio is approximately equal to 2.5 (table 2 ), which is comparable to values measured in the wake of regular grids (e.g., Antonia et al. 2003).

\subsection{Power-law decay}

In a wind tunnel, the temporal decay shown in (1.1) is transformed into spatial decay via $t=x / U$, if $U$ is constant throughout the test-section. The power-law may then be expressed as

$$
\left\langle q^{2}\right\rangle=A\left(\frac{x}{L_{0}}-\frac{x_{0}}{L_{0}}\right)^{m},
$$

where $A$ is a constant of proportionality, $x_{0}$ is a virtual origin, and $L_{0}$ is used to nondimensionalize $x$. Typically, the normalizing length scale for classical grid experiments is chosen to be $M$.

It is common to use a least-squares method to fit empirical measurements of $\left\langle q^{2}\right\rangle$ to (3.4). Mohamed \& LaRue (1990) remarked that the accuracy of the least-squares fitting is greatly improved if the number of variable parameters is reduced. Rather than treating $x_{0}$ as a free parameter, Lavoie et al. (2007) developed a technique where a range of $x_{0}$ values were inserted into the power-law, and $A$ and $m$ were determined with less variance for each $x_{0}$. There is also significant ambiguity associated with identifying $x_{\min }$, which marks the beginning of the power-law decay range (PLDR). Determining $x_{\text {min }}$ 


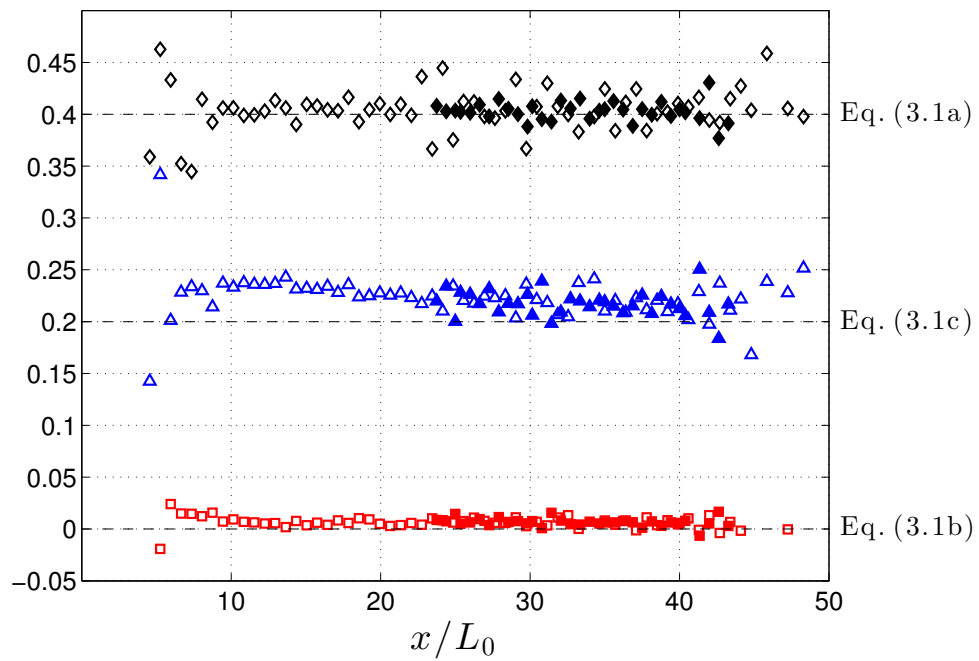

FiguRE 4. Corrsin (1963) streamwise homogeneity criteria; $(\diamond)(3.1 a)$ with +0.4 offset, $(\square)$ $(3.1 b),(\triangle)(3.1 c)$ with +0.2 offset. $R e_{L_{0}}=57,000$ (filled symbols), $R e_{L_{0}}=65,000$ (empty symbols).

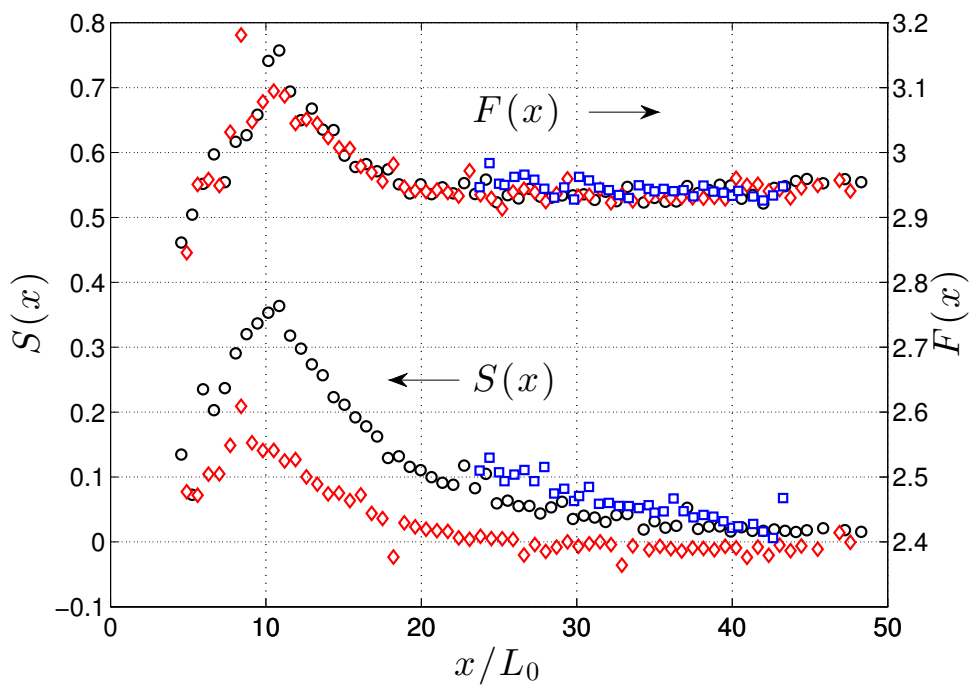

FiguRE 5. Streamwise skewness and flatness; $(\bigcirc) u$-statistics at $\operatorname{Re}_{L_{0}}=65,000,(\diamond)$ $v$-statistics at $R e_{L_{0}}=65,000,(\square) u$-statistics at $R e_{L_{0}}=57,000$.

has been addressed in several ways in previous studies, but typically it is accepted that measurements recorded in the range $x / M \gtrsim 30$ are within the PLDR (Corrsin 1963; Comte-Bellot \& Corrsin 1966; Lavoie et al. 2007; Krogstad \& Davidson 2011).

The technique used here is a combination of that proposed by Lavoie et al. (2007) and the regression technique of Krogstad \& Davidson (2011). The present technique is aimed at reducing ambiguities associated with user choice in the power-law fitting process. It is as follows:

(a) A linear fit using a least-squares regression algorithm is made to the natural logarithm of (3.4) for virtual origins over a range $-8.0 \leqslant x_{0} / L_{0} \leqslant 13.0$ in increments of 


\begin{tabular}{c|ccccccccc}
\hline $\begin{array}{c}\text { Fit } \\
\#\end{array}$ & $\begin{array}{c}U \\
{[\mathrm{~m} / \mathrm{s}]}\end{array}$ & $R e_{L_{0}}$ & $\begin{array}{c}\text { Fit range } \\
{\left[x / L_{0}\right]}\end{array}$ & $x_{\min } / L_{0}$ & $x_{0} / L_{0}$ & $m$ & $m_{u}$ & $m_{v}$ & $\begin{array}{c}\chi \\
{[\%]}\end{array}$ \\
\hline 1 & 9.0 & 57,000 & {$[23.7,44.6]$} & 24.1 & +6.0 & -1.39 & -1.32 & -1.44 & 1.11 \\
2 & 10.6 & 65,000 & {$[20.3,48.5]$} & 23.8 & +7.0 & -1.37 & -1.39 & -1.36 & 0.77 \\
3 & 10.6 & 65,000 & {$[3.5,20.0]$} & 8.8 & -8.0 & -2.79 & -3.45 & -2.58 & 0.89
\end{tabular}

TABLE 3. Power-law fitting parameters and results.

0.5 . For each $x_{0} / L_{0}$, the power-law is also estimated for various $x_{\min } / L_{0}$ values starting from the first measurement location. Fits are not made to ranges smaller than $10 L_{0}$ due to a rapid decrease in fitting accuracy. Through this process, a matrix of $m$ values is generated where one dimension represents the dependence of $m$ on $x_{0} / L_{0}$ and the other on $x_{\min } / L_{0}$.

(b) The virtual origin is selected by choosing the $x_{0} / L_{0}$ which yields the lowest standard deviation of $m$ relative to its mean for all choices of $x_{\min } / L_{0}$. This has the effect of choosing the $x_{0} / L_{0}$ which is least influenced by the PLDR, indicating that the power-law is constant over the largest downstream range.

(c) Given $x_{0} / L_{0}$ from step $(b)$, the normalized root-mean-square deviation $(\chi)$ is then calculated between the data and the power-law fit for each possible choice of $x_{\min } / L_{0}$. $x_{\min } / L_{0}$ is then chosen such that $\chi$ is minimized. Through this process, the PLDR represents the power-law range that fits the data most accurately.

$(d)$ The percentage difference $\left(\Delta_{i}\right)$ is then calculated at each measurement location between $\left\langle q^{2}\right\rangle$ and the power-law fit obtained from step $(c)$. Steps $(a),(b)$, and $(c)$ are then repeated iteratively, excluding from the fitting process any locations $i$ where $\Delta_{i}$ is outside of two standard deviations from its mean. The end criterion is that no outliers are detected.

The above technique was applied to the streamwise measurements of $\left\langle q^{2}\right\rangle$ for the square-fractal-element grid at both $R e_{L_{0}}$. In general, the process converged in two iterations and discarded less than $4 \%$ of the streamwise measurements. Figure 6 shows the variation of $m$ with $x_{\min } / L_{0}$ for different values of $x_{0} / L_{0}$ for the $R e_{L_{0}}=65,000$ data, which is representative of both cases. The error bars indicate the value of $\chi$ for each fit. The uncertainties on the power-law parameters are $x_{0} / L_{0} \pm 2, x_{\min } / L_{0} \pm 1$, and $m \pm 0.1$. These relatively high uncertainties compared to previous regular grid experiments are attributable to the relatively short decay range investigated here. Table 3 shows the results of the power-law fitting process. The $R e_{L_{0}}=57,000$ data were acquired only in the grid far-field, and as such only one fit to this data is performed, while the $R e_{L_{0}}=65,000$ data were acquired over an extended range, and fits are made to both the near-grid and far-field regions.

The regions identified for fitting of the $R e_{L_{0}}=65,000$ data were: $x / L_{0} \leqslant 20$, to compare results directly to previous fractal work; and $x / L_{0}>20$, the region that is approximately homogeneous and isotropic as identified in section 3.1. While there is no theoretical expectation for the presence of a power-law in the inhomogenous region of the flow, a power-law with $m=-2.79$ fits the present data in the near-grid region. This exponent is similar to the $m \approx-2.5$ observed by Valente \& Vassilicos (2011) in the same region relative to $L_{0}$. The near-grid decay of $\left\langle q^{2}\right\rangle$ at $R e_{L_{0}}=65,000$ is shown in figure 7 (a) with fit \#3 from table 3 applied. The figure shows that the fit only applies for $x / L_{0} \lesssim 20$ and deviates rapidly from the trend described by the data in the far-field. 


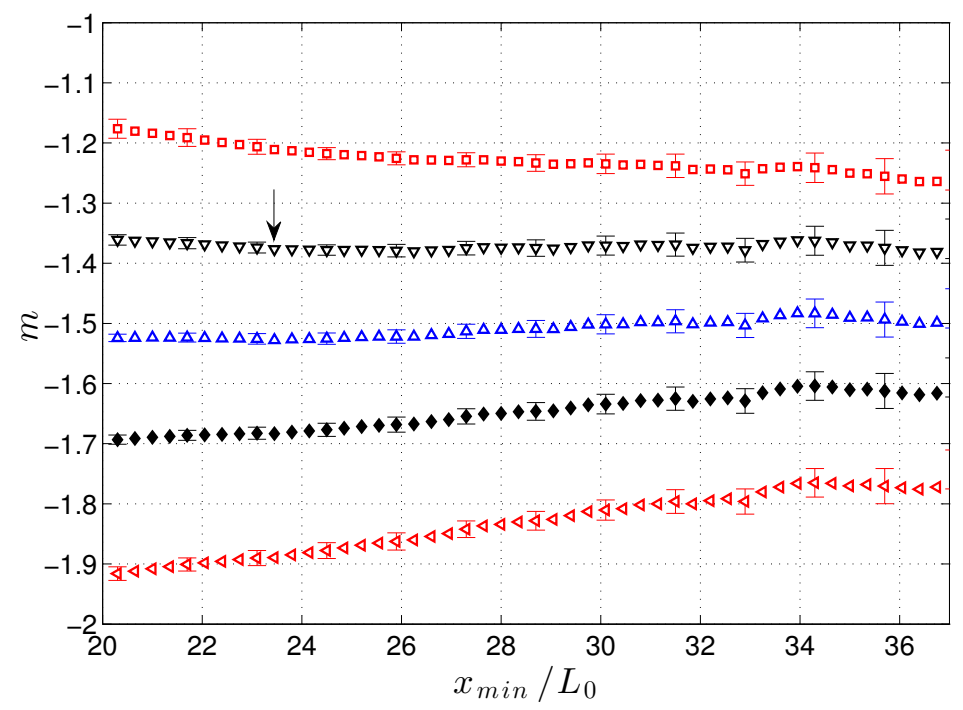

FiguRE 6. Variations of $m$ with $x_{\min } / L_{0}$ for the fit to the $R e_{L_{0}}=65,000$ far-field data; $(\square) x_{0} / L_{0}=10,(\nabla) x_{0} / L_{0}=7,(\triangle) x_{0} / L_{0}=4,(\triangleleft) x_{0} / L_{0}=1,(\triangleleft) x_{0} / L_{0}=-3$. An arrow indicates the algorithm's chosen solution.

On the other hand, fit \#2 with $m=-1.37$ is more accurate in the far-field, as shown in figure 7(b). Fit \#1, applied to the far-field of the $R e_{L_{0}}=57,000$ case provides a decay exponent of $m=-1.39$, nearly identical to the higher $R e_{L_{0}}$ case. These results highlight that the turbulent kinetic energy decays at different rates in the near- and far-fields.

To gain confidence in the estimate of $m$, it is common to estimate the power-law based on $\left\langle u^{2}\right\rangle$ and $\left\langle v^{2}\right\rangle$ independently, using the values of $x_{0} / L_{0}$ and $x_{\min } / L_{0}$ found for $\left\langle q^{2}\right\rangle$. This also ensures that the results are self-consistent. Table 3 provides values of $m_{u}$ and $m_{v}$ based on the decay of $\left\langle u^{2}\right\rangle$ and $\left\langle v^{2}\right\rangle$, respectively. Values of $m_{u}$ and $m_{v}$ for fits \#1 and $\# 2$ are within $\pm 5 \%$ and the uncertainty of $m$ estimated from $\left\langle q^{2}\right\rangle$. Fit \#3, which was made in the inhomogeneous $x / L_{0} \leqslant 20$ region, does not have as strong agreement between $m, m_{u}$ and $m_{v}$.

The validity of using a power-law to fit the decay of $\left\langle q^{2}\right\rangle$ can be verified through the evolution of the Taylor microscale. Given (2.1) and (3.2), it follows that the streamwise evolution of the Taylor microscale is linear with $x$, viz.

$$
\lambda^{2}=\frac{10 \nu}{m U}\left(x-x_{0}\right)
$$

in the region where $\left\langle q^{2}\right\rangle$ is described by a power-law. Figure 8 demonstrates very good agreement between the measured values of $\lambda^{2}$ and (3.5) evaluated with the values of $m$ and $x_{0}$ obtained from fitting the power-law to $\left\langle q^{2}\right\rangle$, which are given in table 3 . In figure 8 , there are two distinct regions in the evolution of $\lambda^{2}$. These two regions and the location of the transition between them are consistent with the two decay regions identified from the evolution of $\left\langle q^{2}\right\rangle$.

Significantly, the values of $m$ found in the far-field for the present data are in agreement with the regular grid results reported in the literature. Furthermore, $x_{\min } / L_{0}=23.6$ and 24.1 for $R e_{L_{0}}=65,000$ and 57,000, respectively, are also in good agreement with typical values of $20 \lesssim x_{\min } / M \lesssim 35$ for regular grids if $L_{0}$ is assumed to be equivalent to $M$ (Comte-Bellot \& Corrsin 1966; Lavoie et al. 2007; Krogstad \& Davidson 2011). These 


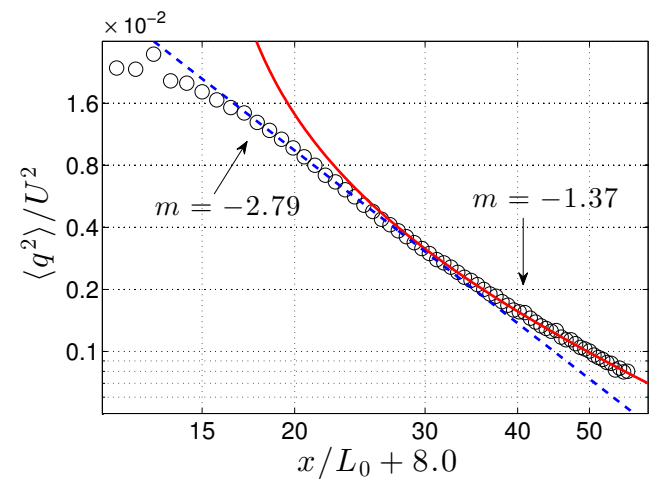

(a)

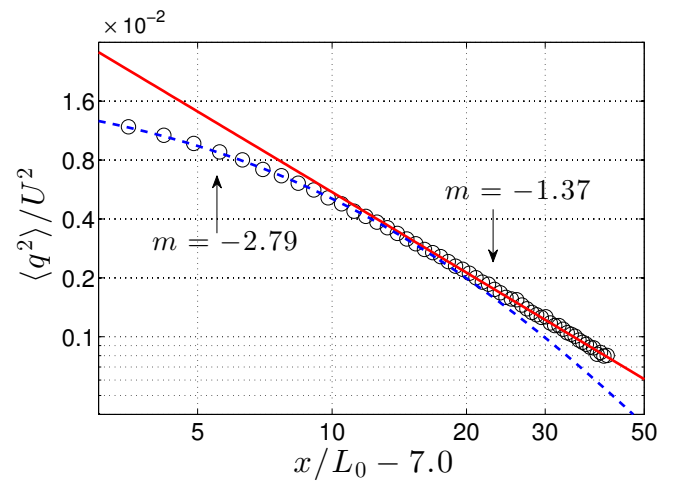

(b)

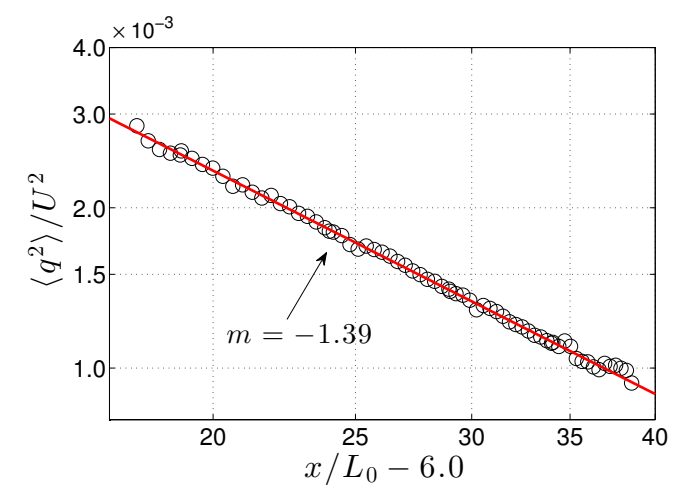

(c)

FiguRE 7 . Decay of the turbulent kinetic energy, $\left\langle q^{2}\right\rangle$, plotted vs. $\left(x-x_{0}\right) / L_{0}$ with the fits from table 3 for (a) the near-field at $R e_{L_{0}}=65,000$, (b) the far-field at $R e_{L_{0}}=65,000$, and (c) the far-field at $R e_{L_{0}}=57,000$. Only every second point is plotted for the $R e_{L_{0}}=65,000$ data in order to reduce clutter.

values of $x_{\min } / L_{0}$ are beyond the farthest downstream point of previous fractal grid measurements.

\subsection{Self-similarity of the spectra}

The spectra $\left(\left\langle u^{2}\right\rangle=\int_{0}^{\infty} F_{11}(k) \mathrm{d} k\right)$ measured at different decay time in HIT are expected to only collapse at the large scales when normalized with $L_{u}$ and $\left\langle u^{2}\right\rangle$, which are referred to as large scale (or outer) variables. The opposite is true when normalized by Kolmogorov (or inner) variables, $\eta$ and $\nu$ (e.g., Antonia \& Orlandi 2004). Mazellier \& Vassilicos (2010) and Valente \& Vassilicos (2011) measured anomalous behaviour in the wake of their square fractal grid. In particular, they found that normalization by $L_{u}$ and $\left\langle u^{2}\right\rangle$ approximately collapsed the spectra at all wavenumbers. This was linked to an approximate proportionality of $L_{u}$ and $\lambda$.

Figure 9 shows the far- and near-field spectra under three different normalizations. Figure 9 (a) demonstrates that when scaled by Kolmogorov variables, the far-field spectra are collapsed only at high wavenumbers, as expected. Similarly, figure 9(c) shows that in the far-field, large scale variables collapse the spectra only at low wavenumbers. This is classical Richardson-Kolmogorov phenomenology and agrees with typical decaying HIT results (e.g., Antonia \& Orlandi 2004; Krogstad \& Davidson 2011). Figure 9(b) demonstrates that under Taylor variable normalization the spectra is approximately collapsed 


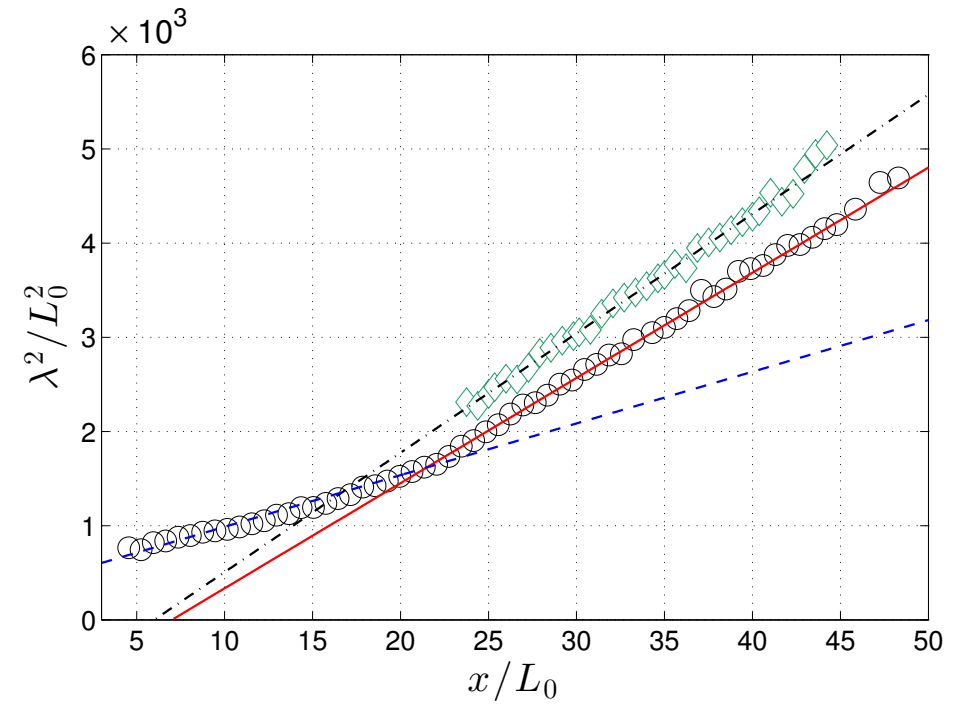

Figure 8. Evolution of the Taylor microscale, $\lambda^{2}$, with fits to (3.5) using values of $m$ and $x_{0}$ from table $3 ;(\bigcirc) R e_{L_{0}}=65,000,(\diamond) R e_{L_{0}}=54,000$. Only every second point is plotted in order to reduce clutter.

at all scales, also in good agreement with previous grid turbulence results (Antonia et al. 2003; Valente \& Vassilicos 2011).

Interestingly, figure 9(d) shows that the spectra are approximately collapsed at all scales in the near-grid region when normalized by large scale variables. This agrees with the results of Vassilicos and co-workers. Figure 10 shows the evolution of $L_{u} / \lambda$ in the present flow. Two distinct behaviours of $L_{u} / \lambda$ are apparent in figure 10(b). In the farfield, there is good agreement with $R e_{\lambda} \propto L_{u} / \lambda$ (Tennekes \& Lumley 1972); the range of $R e_{\lambda}$ here is generated by the downstream evolution of the turbulence. However, in the near-field, the dependence of $L_{u} / \lambda$ on $R e_{\lambda}$ wanes (if one neglects the points $x / L_{0} \leqslant 8.8$, which are measured very close to the grid where the flow has not organized itself from distinct wakes into homogeneous turbulence). In the near-field, $L_{u} / \lambda$ is approximately constant, similar to the results of Valente \& Vassilicos $(2011,2012)$.

\subsection{Normalized energy dissipation rate}

A final note can be made on the scaling of the energy dissipation rate, which is expected to follow $\epsilon \sim\left\langle q^{2}\right\rangle^{3 / 2} / L_{u}$. This is often measured in experiments using the normalized energy dissipation rate,

$$
C_{\epsilon}=\frac{\langle\epsilon\rangle L_{u}}{\left\langle u^{2}\right\rangle^{3 / 2}} .
$$

Following from Richardson-Kolmogorov phenomenology, $C_{\epsilon} \approx$ constant with $x / L_{0}$ in grid turbulence if $R e_{\lambda}$ is adequately high (Sreenivasan 1984; Burattini et al. 2005). However, Valente \& Vassilicos (2011) measured $C_{\epsilon}$ growing from roughly 0.5 to 0.7 for space-filling square fractals and linked this to the approximate constancy of $L_{u} / \lambda$ and a possible breakdown of the classical $\epsilon \sim\left\langle q^{2}\right\rangle^{3 / 2} / L_{u}$ scaling. Figure 11 shows $C_{\epsilon}$ estimated using $\langle\epsilon\rangle_{d}$ for both $R e_{L_{0}}$. In this flow, $C_{\epsilon}$ grows in the near-grid region and becomes approximately constant for $x / L_{0}>25$. The $C_{\epsilon}$ results from figure 7 of Valente \& Vassilicos (2011) have been digitized and are shown in figure 11 here for comparison. The present 


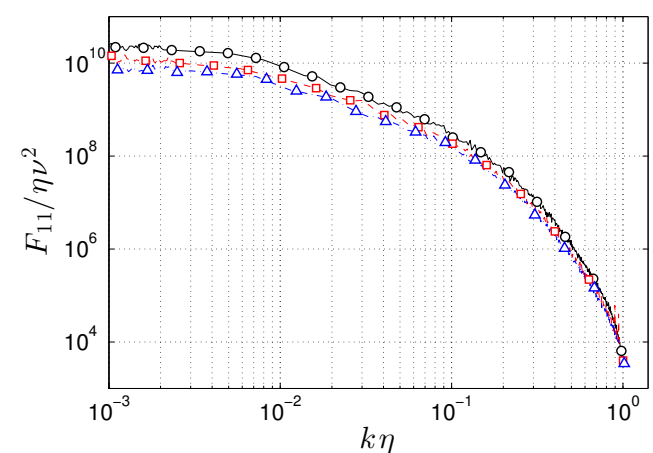

(a)

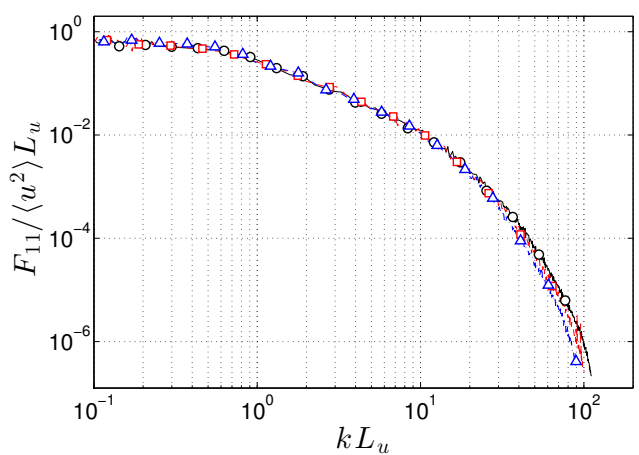

(c)

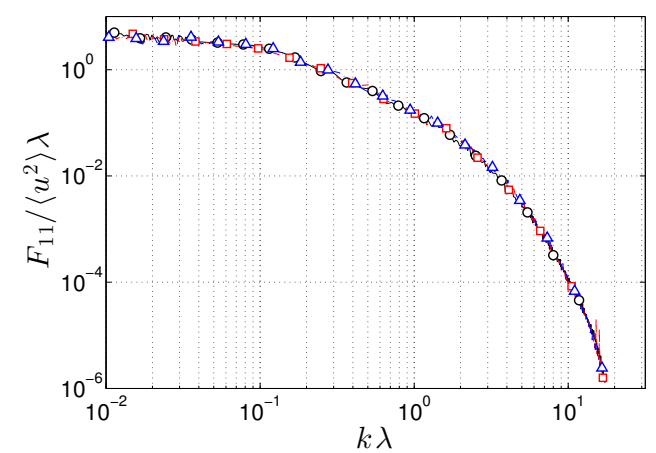

(b)

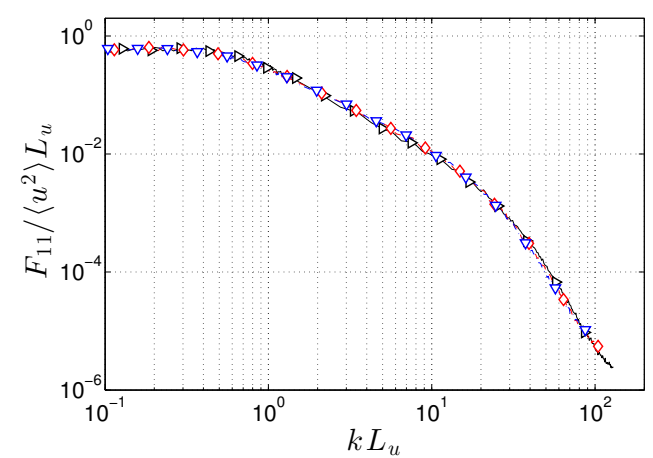

(d)

FiguRE 9. Spectra measured in the grid far-field for $R e_{L_{0}}=57,000$, normalized by (a) Kolmogorov variables, (b) Taylor variables, and (c) large scale variables; $(\bigcirc) x / L_{0}=25.0,(\square)$ $x / L_{0}=35.0,(\triangle) x / L_{0}=44.6$. (d) Spectra measured in the near-grid region for $R e_{L_{0}}=65,000$ normalized by large scale variables; $(\triangleright) x / L_{0}=10.2,(\diamond) x / L_{0}=15.1,(\nabla) x / L_{0}=20.0$.

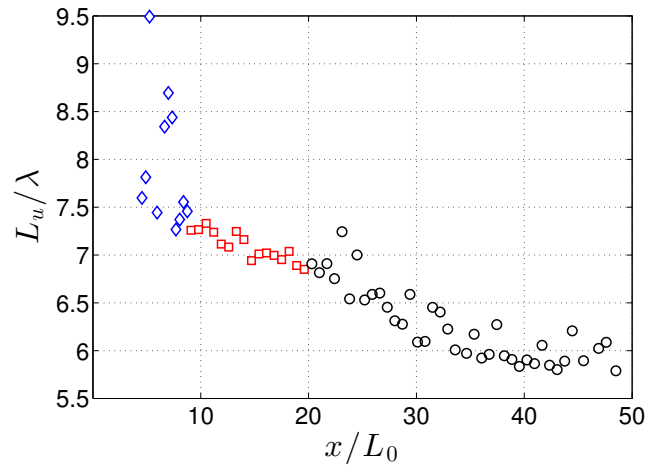

(a)

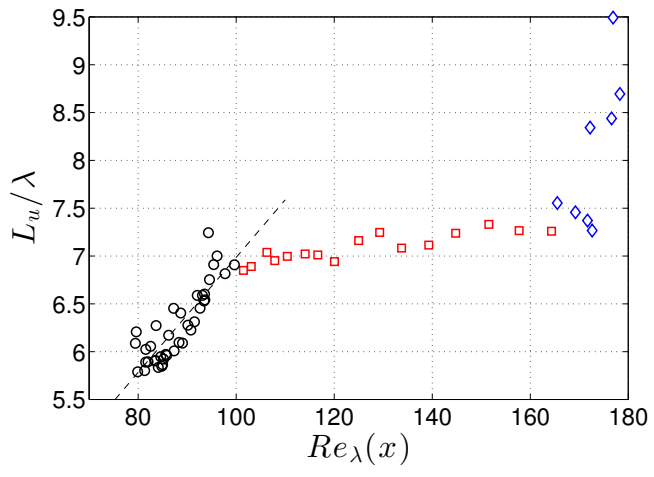

(b)

FiguRE 10. Evolution of $L_{u} / \lambda$ for $R e_{L_{0}}=65,000$ with (a) $x / L_{0}$, and (b) $R e_{\lambda}$ with linear fit in far-field representing $R e_{\lambda} \propto L_{u} / \lambda$; $(\diamond) x / L_{0} \leqslant 8.8$, (口) $8.8<x / L_{0}<20$, (O) $x / L_{0} \geqslant 20$. Only every second point is plotted in order to reduce clutter. 


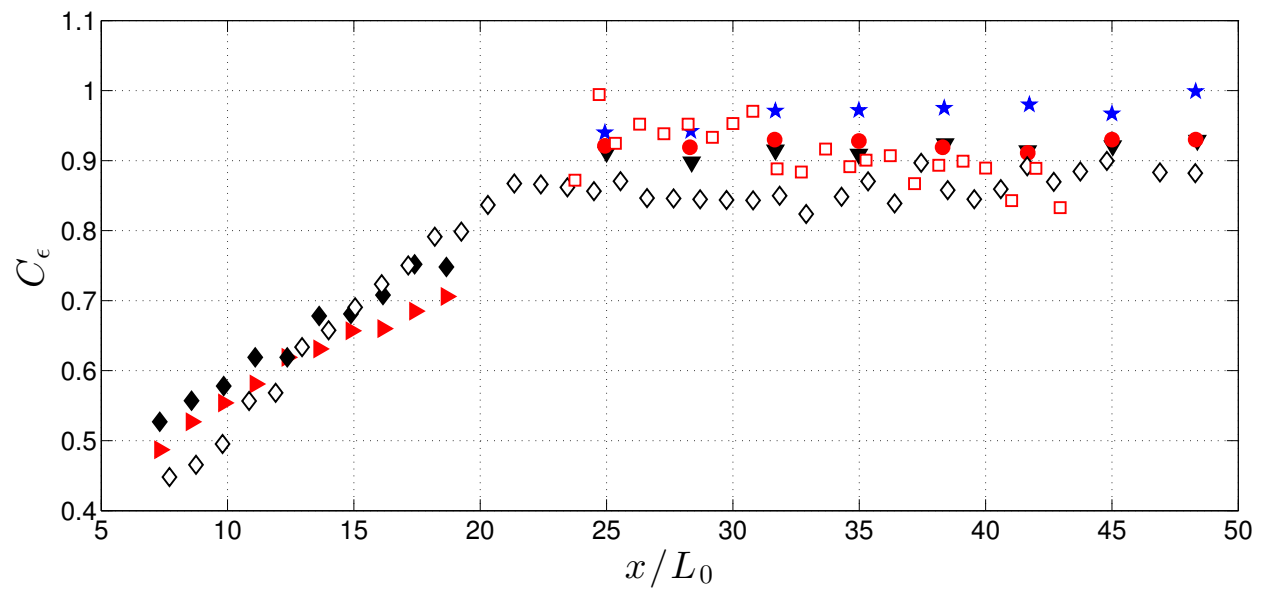

Figure 11. Normalized energy dissipation rate, $C_{\epsilon}$, for the present data (open symbols), only every third point is plotted to reduce clutter; $(\diamond) R_{L_{0}}=65,000,(\square) R e_{L_{0}}=57,000$. Digitized $C_{\epsilon}$ data from Valente \& Vassilicos (2011) (closed symbols) for a Fractal Square Grid (FSG), $L_{0}=237.8 \mathrm{~mm}$, and a Regular Grid $(\mathrm{RG}), L_{0}=60.0 \mathrm{~mm} ;(\bullet) \mathrm{FSG}$ at $U=10 \mathrm{~m} / \mathrm{s},(\diamond)$ FSG at $U=15 \mathrm{~m} / \mathrm{s},(\star) \mathrm{RG}$ at $U=10 \mathrm{~m} / \mathrm{s},(\boldsymbol{\nabla}) \mathrm{RG}$ at $U=15 \mathrm{~m} / \mathrm{s},(\bullet) \mathrm{RG}$ at $U=20 \mathrm{~m} / \mathrm{s}$.

measurements reconcile previous regular grid observations that $C_{\epsilon}$ is constant adequately far from the grid, and previous fractal measurements that show $C_{\epsilon}$ may increase rapidly near the grid. These two regions also appear to correlate with the two distinct slope regions of $L_{u} / \lambda$ in figure $10(\mathrm{~b})$ and the start of the homogeneous region of the flow. Interestingly, in the near-field of a regular grid, Valente \& Vassilicos (2012) observed rapid growth of $C_{\epsilon}$ becoming constant sufficiently far downstream (see figure 6 of their work), suggesting this $C_{\epsilon}$ phenomenon may be more general than originally suspected.

\section{Conclusions}

A square-fractal-element grid was designed to reduce the size of the largest element of the grid relative to the wind tunnel dimensions, enabling observations farther downstream than attainable by previous studies. The fractal grid consists of several small square fractal elements mounted to a background mesh. Measurements were conducted at $R e_{L_{0}}=57,000$ and 65,000 over the range $3.5 \leqslant x / L_{0} \leqslant 48.5$. It was found that a classical power-law decay region does exist adequately far from the grid. Power-law exponents of $m=-1.37$ and -1.39 were estimated based on $\left\langle q^{2}\right\rangle$ for $R e_{L_{0}}=65,000$ and 57,000 , respectively. The classical power-law decay region was found to begin at $\sim 24 L_{0}$. These results, both for $m$ and the start of this decay region, are in good agreement with regular grid turbulence results from the literature if $L_{0}$ is taken to be roughly equivalent to $M$. When a power-law was fit to the near-grid region, $x / L_{0} \leqslant 20, m=-2.79$ was observed, which is similar to the $m \approx-2.5$ result of Valente \& Vassilicos (2011). This near-grid region was characterized by poor transverse homogeneity of the mean flow and turbulent quantities, which appears to contrast with earlier fractal grid measurements. Significantly, it was shown that two distinct region with different turbulence decay rates exist.

It was also shown that when normalized by large scale variables the far-field turbulent spectra are collapsed only at low wavenumbers; similarly, normalization of the far-field spectra by Kolmogorov variables results in collapse only at the small scales. In general, the 
far-field is in good agreement with results expected from classical Richardson-Kolmogorov phenomenology, including $C_{\epsilon} \approx$ constant and $L_{u} / \lambda \propto R e_{\lambda}$. However, the near-field was found to agree with Valente \& Vassilicos (2011), i.e. large scale variables collapse the spectra at all wavenumbers, $C_{\epsilon}$ increases rapidly, and $L_{u} / \lambda$ changes slowly. Given the results of Valente \& Vassilicos (2012), who found that $C_{\epsilon}$ also increases rapidly in the near-grid region of a regular grid, the present results suggest that the $C_{\epsilon}$ behaviour for the square-fractal-element grid and a regular grid is the same if measurements are conducted over a comparable range relatively to $L_{0}$.

The major conclusion of this work is that the turbulence close to the square-fractalelement grid behaves in a manner similar to that observed in previous fractal studies, while the turbulence far downstream behaves as one might expect from a regular grid. This suggests that, while it may extend the mixing region, the fractal nature of the grid does not produce fundamentally different turbulence from previous grid experiments, provided the turbulence has had an opportunity to fully develop some distance downstream.

The authors would like to acknowledge the financial support of the Natural Sciences and Engineering Research Council of Canada (NSERC), and the valiant efforts of Mr. R. Santos Baptista and Mr. J. Rajasekaran who aided in preparing the squarefractal-element grid for experimentation.

\section{REFERENCES}

Antonia, R. A. \& Orlandi, P. 2004 Similarity of decaying isotropic turbulence with a passive scalar. J. Fluid Mech. 505 (123-151).

Antonia, R. A., Smalley, R. J., Zhou, T., Anselmet, F. \& Danaila, L. 2003 Similarity of energy structure functions in decaying homogeneous isotropic turbulence. J. Fluid Mech. 487, 245-269.

Antonia, R. A., Zhou, T. \& Zhu, Y. 1998 Three-component vorticity measurements in a turbulent grid flow. J. Fluid Mech. 374, 29-57.

Antonia, R. A., Zhu, Y. \& Kim, J. 1994 Corrections for spatial velocity derivatives in a turbulent shear flow. Exp. Fluids 16, 411-413.

BAtChelor, G. K. 1948 Energy decay and self-preserving correlation functions in isotropic turbulence. Q. Appl. Maths 6, 97-116.

Benedict, L. H. \& Gould, R. D. 1996 Towards better uncertainty estimates for turbulence statistics. Exp. Fluids 22, 129-136.

Burattini, P. \& Antonia, R. A. 2005 The effect of different X-wire calibration schemes on some turbulence statistics. Exp. Fluids 38, 80-89.

Burattini, P., Lavoie, P. \& Antonia, R. A. 2005 On the normalized turbulent energy dissipation rate. Phys. Fluids 17 (098103).

Comte-Bellot, G. \& Corrsin, S. 1966 The use of a contraction to improve the isotropy of grid-generated turbulence. J. Fluid Mech. 25 (4), 657-682.

Corrsin, S. 1963 Turbulence: experimental methods. In Handbuch der Physik (ed. S. Flügge \& C.A. Truesdell), pp. 524-89. Springer.

Elsner, J. W. \& ElsneR, W. 1996 On the measurement of turbulence energy dissipation. Meas. Sci. Tech. 7, 1334-1348.

Ewing, D. \& George, W. K. 2000 The effect of cross-flow velocity on mean-square derivatives measured using hot wires. Exp. Fluids 25 (5), 418-428.

George, W. K. 1992 The decay of homogeneous isotropic turbulence. Phys. Fluids 4 (7), 1492-1509.

George, W. K. \& Hussein, H. J. 1991 Locally axisymmetric turbulence. J. Fluid Mech. 233, $1-23$.

GaD-El HaK, M. \& Corrsin, S. 1974 Measurements of the nearly isotropic turbulence behind a uniform jet grid. J. Fluid Mech. 62, 115-143. 
Hearst, R. J., Buxton, O. R. H., Ganapathisubramani, B. \& Lavoie, P. 2012 Experimental estimation of fluctuating velocity and scalar gradients in turbulence. Exp. Fluids 53, 925-942.

Hultmark, M. \& Smits, A.J. 2010 Temperature corrections for constant temperature and constant current hot-wire anemometers. Meas. Sci. Tech. 21 (105404).

Hurst, D. \& VAssilicos, J. C. 2007 Scalings and decay of fractal-generated turbulence. Phys. Fluids 19 (035103).

von KÁrmán, T. \& Howarth, L. 1938 On the statistical theory of isotropic turbulence. Proc. R. Soc. London, Ser. A 164, 192-215.

Krogstad, P.-A. \& Davidson, P. A. 2010 Is grid turbulence Saffman turbulence? J. Fluid Mech. 642, 373-394.

Krogstad, P.-A. \& Davidson, P. A. 2011 Freely decaying, homogeneous turbulence generated by multi-scale grids. J. Fluid Mech. 680, 417-34.

Krogstad, P.-A. \& Davidson, P. A. 2012 Near-field investigation of turbulence produced by multi-scale grids. Phys. Fluids 24 (035103).

Lavoie, P., Duenidi, L. \& Antonia, R. A. 2007 Effects of initial conditions in decaying turbulence generated by passive grids. J. Fluid Mech. 585, 395-420.

Mazellier, N. \& Vassilicos, J. C. 2010 Turbulence without Richardson-Kolmogorov cascade. Phys. Fluids 22 (075101).

Meyers, J. \& Meneveau, C. 2008 A functional form for the energy spectrum parametrizing bottleneck and intermittency effects. Phys. Fluids 20 (065109).

Mi, J., Deo, R. C. \& Nathan, G. J. 2005 Fast-convergence iterative scheme for filtering velocity signals and finding Kolmogorov scales. Phys. Rev. E $\mathbf{7 1}$ (066304).

Mi, J., Xu, M. \& Du, C. 2011 Digital filter for hot-wire measurements of small-scale turbulence properties. Meas. Sci. Tech. 22 (125401).

Miller, I. S., Shah, D. A. \& Antonia, R. A. 1987 A constant temperature hot-wire anemometer. J. Phys. E: Sci. Instrum. 20, 311-314.

Mohamed, M.S. \& LaRue, J.C. 1990 The decay power law in grid-generated turbulence. J. Fluid Mech. 219, 195-214.

SAfFman, P. J. 1967 The large-scale structure of homogeneous turbulence. J. Fluid Mech. 27, 581-593.

SReenivasan, K. R. 1984 On the scaling of the turbulence energy dissipation rate. Phys. Fluids 27 (5), 1048-1051.

Tennekes, H. \& Lumley, J. L. 1972 A First Course in Turbulence. MIT Press.

Valente, P. C. \& Vassilicos, J. C. 2011 The decay of turbulence generated by a class of multiscale grids. J. Fluid Mech. 687, 300-340.

Valente, P. C. \& Vassilicos, J. C. 2012 Universal dissipation scaling for nonequilibrium turbulence. Phys. Rev. Lett. 108 (214503).

Wang, H. \& George, W. K. 2002 The integral scale in homogeneous isotropic turbulence. J. Fluid Mech. 459, 429-443.

WyngaARD, J. C. 1968 Measurements of small-scale turbulence structure with hot wires. J. Sci. Instr. 1 (2), 1105-1108.

Zhu, Y. \& Antonia, R. A. 1995 a Effect of wire separation on X-probe measurements in a turbulent flow. J. Fluid Mech. 287, 199-223.

Zhu, Y. \& Antonia, R. A. $1995 b$ The spatial resolution of two X-probes for velocity derivative measurements. Meas. Sci. Tech. 6, 538-549.

Zhu, Y. \& Antonia, R. A. 1996 The spatial resolution of hot-wire arrays for the measurement of small-scale turbulence. Meas. Sci. Tech. 7, 1349-1359. 